\title{
Correction to: Procalcitonin levels in candidemia versus bacteremia: a systematic review
}

\author{
Andrea Cortegiani ${ }^{1 *} \mathbb{D}$, Giovanni Misseri ${ }^{1}$, Mariachiara Ippolito ${ }^{1}$, Matteo Bassetti ${ }^{2}$, Antonino Giarratano ${ }^{1}$, \\ Ignacio Martin-Loeches ${ }^{3,4}$ and Sharon Einav ${ }^{5}$
}

\section{Correction to: Crit Care (2019)23:190 https://doi.org/10.1186/s13054-019-2481-y}

The author wish to note there are three imprecisions in the article [1]. Part of the sentence "All the studies identified were retrospective, except for three prospective and one secondary analysis of a prospectively collected dataset" was omitted, leading to the declaration that "...the studies identified were all retrospective, except for one secondary analysis of a prospective dataset" in the "Results" of the abstract and "All studies were retrospective, except for one secondary analysis of a prospectively collected dataset" under the subheading "Characteristics of the included studies". Table 1 reports the correct number of prospective analyses. Also, the number of excluded records in Fig. 1 should be 1132 and not 1136. Finally, the procalcitonin (PCT) value in the Gram + group in the paper by Basetti et al. [2] should be $8.9 \pm 7.5$ in Table 1. This has now been included in this correction article.

\section{Abbreviation}

PCT: Procalcitonin

\section{Acknowledgments}

None.

\section{Authors' contributions}

AC, MI, SE, checked the data and draft the manuscript. All authors read and approved the final correction statement.

\section{Funding}

None.

\section{Availability of data and materials}

All related data are reported in the text or in additional filies.
Ethics approval and consent to participate Not applicable.

\section{Consent for publication}

Not applicable.

\section{Competing interests}

IML: Lectures: Thermofisher, Polyphor, J\&J, Virogates, MSD; Advisory board: Fresenius Kabi, MaaT Pharma, Bayer, Gilead, Clinigen, Biotest, Accelerate (all unrelated to the present work). AG: received fees for consultancies or lectures from Orion, Pfizer, MSD (all unrelated to the present work). MB: has received funding for scientific advisory boards, travel and speaker honoraria from Angelini, AstraZeneca, Bayer, Biomerieux, Cidara, Cubist, Gilead, Pfizer, Melinta Therapeutics, Menarini, MSD, Nabriva, Paratek, Roche, Shionogi, Tetraphase, The Medicines Company and Astellas Pharma Inc. (all unrelated to the present work). All other authors declare no relevant competing interests.

\section{Author details}

${ }^{1}$ Department of Surgical, Oncological and Oral Science (Di.Chir.On.S.). Section of Anesthesia, Analgesia, Intensive Care and Emergency, Policlinico Paolo Giaccone. University of Palermo, via del vespro 129, 90127 Palermo, Italy. ${ }^{2}$ Infectious Diseases Division, Department of Medicine, University of Udine and Santa Maria della Misericordia University Hospital. Piazzale Santa Maria della Misericordia 15, Udine, Italy. ${ }^{3}$ Multidisciplinary Intensive Care Research Organization (MICRO), St. James's Hospital, Dublin, Ireland. ${ }^{4}$ Hospital Clinic, Universidad de Barcelona, CIBERes, Barcelona, Spain. ${ }^{5}$ Intensive Care Unit of the Shaare Zedek Medical Medical Centre and Hebrew, University Faculty of Medicine, Jerusalem, Israel.

Received: 3 September 2019 Accepted: 3 September 2019 Published online: 11 October 2019

\footnotetext{
References

1. Cortegiani A, Misseri G, Ippolito M, Bassetti M, Giarratano A, Martin-Loeches I, et al. Procalcitonin levels in candidemia versus bacteremia: a systematic review. Crit Care. 2019;23:190. https://doi.org/10.1186/s13054-019-2481-y.

2. Bassetti M, Russo A, Righi E, Dolso E, Merelli M, Cannarsa N, et al. Comparison between procalcitonin and C-reactive protein to predict blood culture results in ICU patients. Crit Care. 2018;22:252. https://doi.org/10. 1186/s13054-018-2183-x.
}

* Correspondence: cortegiania@gmail.com

${ }^{1}$ Department of Surgical, Oncological and Oral Science (Di.Chir.On.S.). Section of Anesthesia, Analgesia, Intensive Care and Emergency, Policlinico Paolo Giaccone. University of Palermo, via del vespro 129, 90127 Palermo, Italy Full list of author information is available at the end of the article 